\title{
Global Integrable Solution for a Nonlinear Functional Integral Inclusion
}

\author{
A. M. A. El-Sayed ${ }^{1}$ and Sh. M. Al-Issa ${ }^{2}$ \\ ${ }^{1}$ Faculty of Science, Alexandria University, Alexandria, Egypt \\ ${ }^{2}$ Faculty of Science, Beirut Arab University, Beirut, Lebanon \\ Correspondence should be addressed to A. M. A. El-Sayed, amasayed@hotmail.com
}

Received 20 August 2009; Accepted 14 October 2009

Copyright () 2010 A. M. A. El-Sayed and Sh. M. Al-Issa. This is an open access article distributed under the Creative Commons Attribution License, which permits unrestricted use, distribution, and reproduction in any medium, provided the original work is properly cited.

We study the global existence of positive integrable solution for the functional integral inclusion of fractional order $x(t) \in p(t)+$ $F_{1}\left(t, I^{\alpha} f_{2}(t, x(\varphi(t))), t \in(0,1), \alpha \geq 0\right.$, where $F_{1}(t, x(t))$ is a set-valued function defined on $(0,1) \times R^{+}$.

\section{Introduction}

Consider the functional integral equation

$$
x(t)=g(t)+f\left(t, \int_{0}^{1} k(t, s) x(\varphi(t))\right) d s, \quad t \in(0,1) .
$$

The authors (see [1]) proved the existence of monotonic integrable solution of (1), where the the function $f(t, x)$ is assumed to be monotonic in both its arguments and satisfies the Caratheodory and growth conditions.

In [2], the author discusses the existence of solution of the functional integral equation

$$
x(t)=u(t)+\int_{0}^{t} k(t, s) f(s, x(\varphi(s))) d s, \quad t \in(0,1) .
$$

In [3], the author omitted the condition of monotonicity of $f(t, x)$ and proved the existence of integrable solution of (1).

Also he proved (see [4]) the existence of integrable solution of the functional integral equation

$$
x(t)=f_{1}\left(t, r \int_{0}^{1} k(t, s) f_{2}(s, x(s)) d s\right), \quad t \in(0,1),
$$

where $f(t, x)$ is assumed be satisfy the Caratheodory and growth conditions.

Consider the functional integral equation

$$
\begin{array}{r}
x(t)=\int_{0}^{t} k_{1}(t, s) f\left(s, \int_{0}^{s} k_{2}(t, \theta) x(\varphi(\theta)) d \theta\right), \\
t \in(0,1) .
\end{array}
$$

The existence of the nonincreasing integrable solution of (4) was studied in [5], where $f(t, x)$ is nondecreasing in both its arguments and satisfies the Caratheodory and growth conditions.

Here we are concerned with the functional integral equation of fractional order

$$
\begin{array}{r}
x(t)=p(t)+f_{1}\left(t, I^{\alpha} f_{2}(t, x(\varphi(t)))\right), \\
t \in(0,1), \quad \alpha \geq 0,
\end{array}
$$

we prove the global existence of positive integrable solution of $(5)$, where $f_{1}(t, x)$ and $f_{2}(t, x)$ satisfy the Caratheodory and growth conditions. As a generalization of our results we study the global existence of positive integrable solution of the nonlinear functional integral inclusion of fractional order

$$
\begin{array}{r}
x(t) \in p(t)+F_{1}\left(t, I^{\alpha} f_{2}(t, x(\varphi(t)))\right), \\
t \in(0,1), \quad \alpha \geq 0,
\end{array}
$$

where the set-valued map $F_{1}:(0,1) \times R^{+} \rightarrow 2^{R^{+}}$has nonempty closed values.

\section{Preliminaries}

Let $L^{1}=L^{1}(I)$ be the class of Lebesgue integrable functions on the interval $I=[a, b], 0 \leq a<b<\infty$, and let $\Gamma(\cdot)$ be the gamma function. 
Definition 1. The fractional integral of the function $f(\cdot) \in$ $L^{1}(I)$ of order $\alpha \in R^{+}$is defined by (cf. [6-9])

$$
I_{a}^{\alpha} f(t)=\int_{a}^{t} \frac{(t-s)^{\alpha-1}}{\Gamma(\alpha)} f(s) d s .
$$

Theorem 1 (nonlinear alternative of Leray-Shauder type [10]). Let $U$ be an open subset of a convex set $D$ in a Banach space $X$. Assume $0 \in U$ and $T \in C(\bar{U}, D)$. Then either

\section{$\left(A_{1}\right) T$ has a fixed point in $\bar{U}$ or}

$\left(A_{2}\right)$ there exists $\gamma \in(0,1)$ and $x \in \partial U$ such that $x=\gamma T x$.

Theorem 2 (Kolmogorov compactness criterion [11]). Let $\Omega \subseteq L^{p}(0,1), 1 \leq P \leq \infty$. If

(i) $\Omega$ is bounded in $L^{p}(0,1)$

(ii) $x_{h} \rightarrow x$ as $h \rightarrow 0$ uniformly with respect to $x \in \Omega$, then $\Omega$ is relatively compact in $L^{p}(0,1)$, where

$$
x_{h}(t)=\frac{1}{h} \int_{0}^{t+h} x(s) d s
$$

\section{Main Results}

In this section we present our main result by proving the global existence of positive solution $x \in L^{1}$ for the functional integral equatoin (1).

To facilitate our discussion, let us first state the following assumptions.

(i) $p \in L^{1}$.

(ii) $f_{i}:(0,1) \times R^{+} \rightarrow R^{+}, i=1,2$, satisfy Caratheodory condition that is, $f_{i}$ are measurabel in $t$ for any $x \in R^{+}$ and continuous in $x$ for almost all $t \in(0,1)$.

There exist four functions $t \rightarrow a_{i}(t), t \rightarrow b_{i}(t)$ such that

$\left|f_{i}(t, x)\right| \leq a_{i}(t)+b_{i}(t)|x|, \quad i=1,2, \forall t \in(0,1), x \in R$,

where $a_{i}(\cdot) \in L^{1}$ and $b_{i}(\cdot)$ are measurable and bounded.

(iii) $\phi:(0,1) \rightarrow(0,1)$ is absolutely continuous, and there exists a constant $M>0$ such that $\varphi^{\prime}(t) \geq M, \forall t \in$ $(0,1)$.

(iv) Assume that every solution $x(\cdot) \in L^{1}$ to the equation

$$
x(t)=\gamma\left(p(t)+f_{1}\left(t, I^{\alpha} f_{2}(t, x(\varphi(t)))\right)\right),
$$

$t \in(0,1), 0<\beta<1, \gamma \in(0,1)$, satisfies $\|x\| \neq r(r>0$ is arbitrary but fixed).

Define the operator $T$,

$T x(t)=p(t)+f_{1}\left(t, I^{\alpha} f_{2}(t, x(\varphi(t)))\right), \quad t \in(0,1)$.

Now, we are in position to formulate and prove our main result.
Theorem 3. Let the assumptions (i)-(iv) satisfied. Then (1) has at least one positive solution $x \in L_{1}$.

Proof. Let $x$ be an arbitrary element in the open set $B_{r}=\{x$ : $\|x\|\langle r, r\rangle 0\}$.

Then from assumptions (i) and (ii), we have,

$$
\begin{aligned}
& \|T x\|=\int_{0}^{1}|(T x)(t)| d t \leq \int_{0}^{1}|p(t)| d t \\
& +\int_{0}^{1}\left|f_{1}\left(t, I^{\alpha} f_{2}(t, x(\varphi(t)))\right)\right| d t \\
& \leq \int_{0}^{1}|p(t)| d t+\int_{0}^{1} a_{1}(t) d t \\
& +\int_{0}^{1} b(t)\left|I^{\alpha} f_{2}(t, x(\varphi(t)))\right| d t \\
& \leq \int_{0}^{1}|p(t)| d t+\int_{0}^{1}\left|a_{1}(t)\right| d t \\
& +\int_{0}^{1}\left|b_{1}(t)\right| I^{\alpha}\left(a_{2}(t)+b_{2}(t)|x(\varphi(t))|\right) d t \\
& \leq\|p\|+\left\|a_{1}\right\|+b_{1} \int_{0}^{1} \int_{0}^{t} \frac{(t-s)^{\alpha-1}}{\Gamma(\alpha)} a_{2}(s) d s d t \\
& +b_{1} \int_{0}^{1} \int_{0}^{t} \frac{(t-s)^{\alpha-1}}{\Gamma(\alpha)}\left|b_{2}(s)\right||x(\varphi(s))| d s d t \\
& \leq\|p\|+\left\|a_{1}\right\|+b_{1} \int_{0}^{1}\left|a_{2}(s)\right| \int_{s}^{1} \frac{(t-s)^{\alpha-1}}{\Gamma(\alpha)} d t d s \\
& +b_{1} b_{2} \int_{0}^{1}|x(\varphi(s))| \int_{s}^{1} \frac{(t-s)^{\alpha-1}}{\Gamma(\alpha)} d t d s \\
& \leq\|p\|+\left\|a_{1}\right\|+b_{1} \int_{0}^{1}\left|a_{2}(s)\right| \frac{(t-1)^{\alpha}}{\Gamma(\alpha+1)} d s \\
& +b_{1} b_{2} \int_{0}^{1}|x(\varphi(s))| \frac{(t-1)^{\alpha}}{\Gamma(\alpha+1)} d s \\
& \leq\|p\|+\left\|a_{1}\right\|+\frac{b_{1}\left\|a_{2}\right\|}{\Gamma(\alpha+1)} \\
& +\frac{b_{1} b_{2}}{\Gamma(\alpha+1) M} \int_{0}^{1}|x(\varphi(s))| \varphi^{\prime}(s) d s \\
& \leq\|p\|+\left\|a_{1}\right\|+\frac{b_{1}\left\|a_{2}\right\|}{\Gamma(\alpha+1)}+\frac{b_{1} b_{2}}{\Gamma(\alpha+1) M} \int_{\varphi(0)}^{\varphi(1)}|x(u)| d u \\
& \leq\|p\|+\left\|a_{1}\right\|+\frac{b_{1}\left\|a_{2}\right\|}{\Gamma(\alpha+1)}+\frac{b_{1} b_{2}}{\Gamma(\alpha+1) M} \int_{0}^{1}|x(u)| d u \\
& \leq\|p\|+\left\|a_{1}\right\|+\frac{b_{1}\left\|a_{2}\right\|}{\Gamma(\alpha+1)}+\frac{b_{1} b_{2}\|x\|}{\Gamma(\alpha+1) M} .
\end{aligned}
$$

Hence the previous inequality means that the operator $T$ maps $B_{r}$ into $L^{1}$. 
Now, we will show that $T$ is compact. To achieve this goal we will apply Theorem 2 . So, let $\Omega$ be a bounded subset of $B_{r}$. Then $T(\Omega)$ is bounded in $L^{1}$; that is, condition (i) of Theorem 2 is satisfied.

It remains to show that $(T x)_{h} \rightarrow T x$ in $L^{1}$ as $h \rightarrow 0$ uniformly with respect to $T x \in \Omega$. We have the following:

$$
\begin{aligned}
\left\|(T x)_{h}-(T x)\right\| \\
=\int_{0}^{1}\left|(T x)_{h}(t)-(T x)(t)\right| d t \\
=\int_{0}^{1}\left|\frac{1}{h} \int_{t}^{t+h}(T x)_{h}(\tau) d \tau-(T x)(t)\right| d t \\
=\int_{0}^{1}\left|\frac{1}{h} \int_{t}^{t+h}\left((T x)_{h}(\tau)-(T x)(t)\right) d \tau\right| d t \\
\leq \int_{0}^{1} \frac{1}{h} \int_{t}^{t+h}|p(\tau)-p(t)| d \tau d t \\
\quad+\int_{0}^{1} \frac{1}{h} \int_{t}^{t+h} \mid f_{1}\left(\tau, I^{\beta} f_{2}(\tau, x(\varphi(\tau)))\right) \\
\quad-f_{1}\left(t, I^{\beta} f_{2}(t, x(\varphi(t)))\right) \mid d \tau d t .
\end{aligned}
$$

Now $f_{1}, f_{2} \in L^{1}$ and $I^{\beta} f_{2} \in L^{1}$, then (cf. [12])

$$
\begin{aligned}
\frac{1}{h} \int_{t}^{t+h} \mid & f_{1}\left(\tau, I^{\beta} f_{2}(\tau, x(\varphi(\tau)))\right) \\
& -f_{1}\left(t, I^{\beta} f_{2}(t, x(\varphi(t)))\right) \mid d \tau \longrightarrow 0 .
\end{aligned}
$$

Moreover, $p(\cdot) \in L^{1}$. So, we have

$$
\frac{1}{h} \int_{t}^{t+h}|p(\tau)-p(t)| d \tau \longrightarrow 0
$$

for a.e $t \in L^{1}$. Therefore, by Theorem 2 we deduce that $T(\Omega)$ is relatively compact; that is, $T$ is compact operator.

Set $U=B_{r}$ and $D=X=L^{1}(0,1)$. Then in the view of assumption (iv) condition $\left(A_{2}\right)$ of Theorem 1 does not hold. Theorem 1 implies that $T$ has a fixed point. This completes the proof.

\section{Integral Inclusion}

Consider now the integral inclusion (2), where $F_{1}:[0,1] \times$ $R^{+} \rightarrow 2^{R^{+}}$has nonempty closed convex values.

As an important consequence of the main result we can present the following

Theorem 4. Let the assumptions of Theorem 3 be satisfied. The multifunction $F_{1}$ satisfies the following assumptions:

(1) $F_{1}(t, x)$ are nonempty, closed and convex for all $(t, x) \in$ $(0,1) \times R^{+}$

(2) $F_{1}(t, \cdot)$ is lower semicontinuous from $R^{+}$into $R^{+}$,

(3) $F_{1}(\cdot, \cdot)$ is measurable,
(4) there exist a function $a \in L_{1}$ and a measurable and bounded function $b$ such that

$$
\left|F_{1}(t, x)\right| \leq a(t)+b(t)|x| \quad \forall t \in(0,1),
$$

Then there exists at least one positive solution $x \in L^{1}$ of the integral inclusion (2).

Proof. By conditions (1)-(4) (see [13-16]) we can find a selection function $f_{1}$ (Caratheodory function) $f_{1}:(0,1) \times$ $R^{+} \rightarrow R^{+}$such that $f_{1}(t, x) \in F_{1}(t, x)$ for all $(t, x) \in(0,1) \times$ $R^{+}$, this function satisfies condition (ii) of Theorem 3 .

Clearly all assumptions of Theorem 3 are hold, then there exists a positive solution $x \in L^{1}$ such that

$x(t)-p(t)=f_{1}\left(t, I^{\beta} f_{2}(t, x(\varphi(t)))\right) \in F_{1}\left(t, I^{\beta} f_{2}(t, x(\varphi(t)))\right)$.

\section{References}

[1] J. Banaś and Z. Knap, "Integrable solutions of a functionalintegral equation," Revista Matemática de la Universidad Complutense de Madrid, vol. 2, no. 1, pp. 31-38, 1989.

[2] J. Banas and W. G. El-Sayed, Solvability of Functional and Integral Equations in Some Classes of Integrable Functions, Politechnika Rzeszowska, Rzeszów, Poland, 1993.

[3] G. Emmanuele, "About the existence of integrable solutions of a functional-integral equation," Revista Matemática de la Universidad Complutense de Madrid, vol. 4, no. 1, pp. 65-69, 1991.

[4] G. Emmanuele, "Integrable solutions of a functional-integral equation," Journal of Integral Equations and Applications, vol. 4, no. 1, pp. 89-94, 1992.

[5] A. M. A. El-Sayed, N. Sherif, and I. Abou El-Farag, "A nonlinear operator functional equation of Volterra type," Applied Mathematics and Computation, vol. 148, no. 3, pp. 665-679, 2004.

[6] I. Podlubny and A. M. A. EL-Sayed, On Two Defintions of Fractional Calculus, Slovak Academy of Sciences, Institute of Experimental Physics, Watsonova, Slovakia, 1996.

[7] I. Podlubny, Fractional Differential Equation, Academic Press, San Diego, Calif, USA, 1999.

[8] K. S. Miller and B. Ross, An Introduction to the Fractional Calculus and Fractional Differential Equations, A WileyInterscience Publication, John Wiley \& Sons, New York, NY, USA, 1993.

[9] S. G. Samko, A. A. Kilbas, and O. I. Marichev, Integrals and Derivatives of Fractional Orders and Some of Their Applications, Nauka i Tekhnika, Minsk, Belarus, 1987.

[10] K. Deimling, Nonlinear Functional Analysis, Springer, Berlin, Germany, 1985.

[11] J. Dungundji and A. Grance, Fixed Piont Theory, Monografie Mathematyczne, PWN, Warsaw, Poland, 1982.

[12] C. Swartz, Measure, Integration and Function Spaces, World Scientific, Singapore, 1994.

[13] A. Bressan and G. Colombo, "Extensions and selections of maps with decomposable values," Studia Mathematica, vol. 90, no. 1, pp. 69-86, 1988.

[14] M. Cichoń, A. M. A. El-Sayed, and A. H. Hussien, "Existence theorems for nonlinear functional integral equations of fractional orders," Commentationes Mathematicae, vol. 41, pp. 59-67, 2001. 
[15] M. Cichoń, "Multivalued perturbations of $m$-accretive differential inclusions in a non-separable Banach space," Commentationes Mathematicae, vol. 32, pp. 11-17, 1992.

[16] D. Repovš and P. V. Semenov, Continuous Selections of Multivalued Mappings, vol. 455 of Mathematics and Its Applications, Kluwer Academic Publishers, Dordrecht, The Netherlands, 1998. 